\title{
Experimental study on high-speed endurance of electric vehicle at normal temperature $\left(25^{\circ} \mathrm{C}\right)$
}

\author{
Chun $\mathrm{Li}^{*}$, Fan Yang, and Zhenchong Wang \\ China Automotive Technology and Research Center Co., Ltd., Tianjin, 300300, China
}

\begin{abstract}
Keywords: electric vehicle, normal temperature, driving range, battery power, descent rate.
\end{abstract}

\begin{abstract}
Electric vehicle[1] endurance has always been a major concern for car buyers. Based on the six conventional electric vehicles selected from the market, the driving range of the chassis dynamometer with the environment warehouse is first carried out under the CLTC-P condition of normal temperature environment, and compared with the vehicle meter-display driving range. After testing the speed of $100 \mathrm{~km} / \mathrm{h}$ of the driving range, the high-speed driving range at normal temperature is obtained, and then compared with the normal temperature driving range and the meter-display driving range, the drop rate of high-speed driving range is obtained. By analyzing and comparing the different test conditions of 6 vehicles, the influence trend of battery quantity, test quality, resistance and driving mode on high-speed driving range is obtained. Allowing consumers to anticipate their travel plans and also provides data for subsequent car companies to improve the quality of electric vehicles.
\end{abstract}

Foreword: With the rapid progress of living standards, cars have become a means of transportation for every household. The following situation is the phenomenon of urban traffic congestion. In order to alleviate this pressure and the protection of the environment, a line-limiting policy was initiated. As for the newly developed electric vehicles, The driving range is generally only 200 or 300 kilometers two or three years ago, which is in the case of not using air conditioning. Consumers will hesitate because the driving mileage is too small to satisfy their own use. but now the driving range has reached 400 to 500 kilometers, coupled with some state subsidy policies. At the same time, it can also properly avoid the line limit, so people's car demands began to transform from the traditional gasoline car to an electric car. Therefore, buying electric vehicles will still face a lot of problems, such as real endurance, value retention rate, etc. The most prominent concern among these problems is endurance. With the increase of electric vehicles, the city's major shopping malls, hotels, and entertainment venues have begun to provide electric vehicle supply stations-charging piles. So there will be no special fear of travel due to the lack of electricity and have to stay on the way. But driving traveling will not only be in the city, but also occasionally driving outside the city. High-speed driving is inevitable, and if there is no general assessment of high-speed endurance, it may result in staying on the highway. In

\footnotetext{
${ }^{*}$ Corresponding author: lichun2017@catarc.ac.cn
} 
this article, six more common electric vehicles were selected. First, the continuous driving range test at room temperature CLTC-P was conducted to obtain the endurance at normal temperature, and then the continuous driving range at a high-speed of $100 \mathrm{~km} / \mathrm{h}$ was tested. By comparison, the endurance of high-speed $100 \mathrm{~km} / \mathrm{h}$ is calculated.

\section{Test equipment and methods}

\subsection{Selection of test samples}

When selecting test samples, the basic standard is a pure electric passenger car (M1 electric vehicle) in the Chinese mainland market, and the sales volume should reach more than 1,000 units. This test selected 6 vehicles from different car companies for comparison.

\subsection{Test equipment}

The test was conducted in a four-wheel-drive drum laboratory with an environmental warehouse. The test equipment is shown in Table 1:

Table 1. Test equipment.

\begin{tabular}{|c|c|c|}
\hline Equipment name & Model & Main performance parameters \\
\hline $\begin{array}{c}\text { Environmental } \\
\text { warehouse }\end{array}$ & WK $763^{\prime} / 40-60 /$ Ro $/ \mathrm{So}$ & $\begin{array}{c}\text { Temperature control range: }-40^{\circ} \mathrm{C} \sim \\
60^{\circ} \mathrm{C} ; \quad \begin{array}{c}\text { humidity control range: } \\
10 \% \sim 95 \% ;\end{array}\end{array}$ \\
\hline $\begin{array}{c}\text { Chassis } \\
\text { dynamometer }\end{array}$ & AVL ROADSIM $48^{\prime \prime}$ & $\begin{array}{c}\text { inertia simulation range: } 450 \mathrm{~kg} \sim \\
6000 \mathrm{~kg} ; \text { maximum test speed: } 250 \mathrm{~km} / \mathrm{h} ;\end{array}$ \\
\hline
\end{tabular}

The picture of the laboratory is shown in Figure 1:

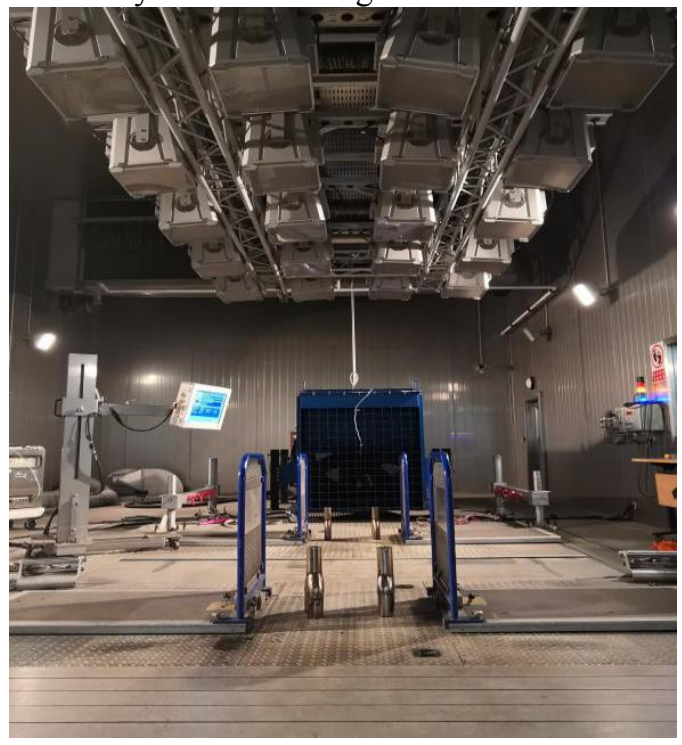

Fig. 1. The real scene of the environmental warehouse.

\subsection{Test method}


The formulation of the test method is mainly based on the "China Electric Vehicle Evaluation (EV-TEST) Management Regulations (2019 Version)". After the initial discharge, the electric vehicle was charged at normal temperature $(25 \pm 5){ }^{\circ} \mathrm{C}$ [2] until it was fully charged and then statically placed in normal temperature environment $(25 \pm 5){ }^{\circ} \mathrm{C}$ for 12-15 hours. At this time, recorded the remaining driving distance of the displayed instrument board. In the next step, continued to work under CLTC-P operating conditions until the driver could not keep up with the operating curve when the accelerator pedal was pressed to the bottom, stopped the test and recorded the driving distance at this time as the driving range at normal temperature. After the previous test was completed, charge it at normal temperature until it was fully charged. After immersing the car for more than 12 hours in the environment of $(25 \pm 5){ }^{\circ} \mathrm{C}$, it is conducting a $100 \mathrm{~km} / \mathrm{h}$ high-speed constant speed endurance test. When the vehicle speed does not reach $90 \mathrm{~km} / \mathrm{h}$, stopped the test and recorded the driving range at this time to be the high-speed driving range at normal temperature.

\subsubsection{Test ambient temperature}

Conduct normal temperature and high speed endurance test at $(25 \pm 5){ }^{\circ} \mathrm{C}$ normal temperature

\subsubsection{Test conditions}

During the test, the most economical driving mode is selected for the driving range test, but the premise is that the vehicle speed in this mode can keep up with the CLTC-P operating curve (the maximum speed $114 \mathrm{~km} / \mathrm{h}$ ) and the high-speed operating condition of $100 \mathrm{~km} / \mathrm{h}$. If it can't keep up with the curve, use other driving modes that can keep up with the curve for testing. The CLTC-P operating condition is one of the cycle conditions of Chinese automobiles, which is suitable for light passenger vehicles in China. The CLTC-P operating condition curve is shown in Figure 2:

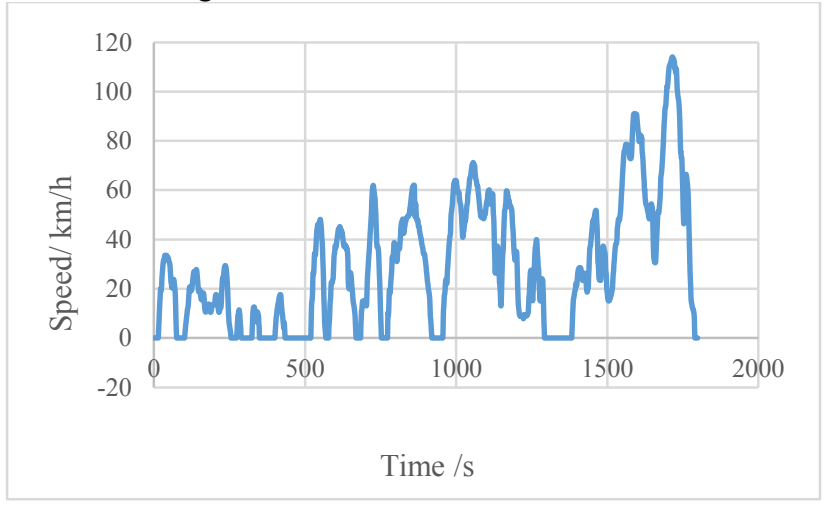

Fig. 2.CLTC-P operating curve.

\subsubsection{End condition of the test}

(A) For the test vehicle with a maximum speed of $114 \mathrm{~km} / \mathrm{h}$ or more, when the driver step on the accelerator pedal to the bottom and cannot keep up with the tolerance range of the CLTC-P curve $( \pm 2 \mathrm{~km} / \mathrm{h})$, the test shall be stopped; (b) For the high-speed constant speed 
test, the test speed of the vehicle is $100 \mathrm{~km} / \mathrm{h}$. And the test should be stopped when the driving speed of the vehicle cannot reach $90 \mathrm{~km} / \mathrm{h}$; (c) When the end condition of the test is reached, the gear is kept in the original driving gear, and the vehicle is allowed to slide to the lowest stable speed before stepping the brake pedal to stop, record the driving distance $(\mathrm{km})$ at this time;

\subsection{Relationship between test quality, battery power and the meter-display driving range}

The test quality refers to the simulated inertia of the chassis dynamometer, which is calculated by the vehicle's curb quality, maximum quality and optional equipment quality[4]. It can be seen from Fig. 3 that the meter-display driving range and the battery power are basically positively correlated, which is indicating that the greater the battery capacity, the longer the theoretical driving distance will be.
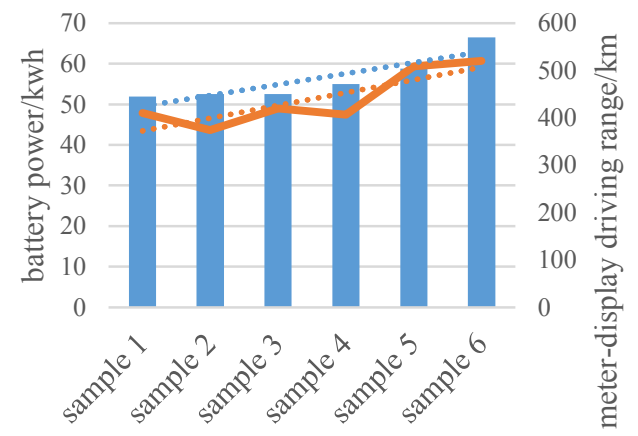

battery power ( kwh)

meter-display driving range $(\mathrm{km})$

Linear(battery power(kwh))

Linear(meter-display driving range $(\mathrm{km}))$

Fig. 3. Comparison of battery power and meter-display driving range.

As can be seen from the figure that the driving range of the six car watch has basically reached more than $350 \mathrm{~km}$, which can already meet the needs of commuting traffic, and short-distance travel is also feasible. 


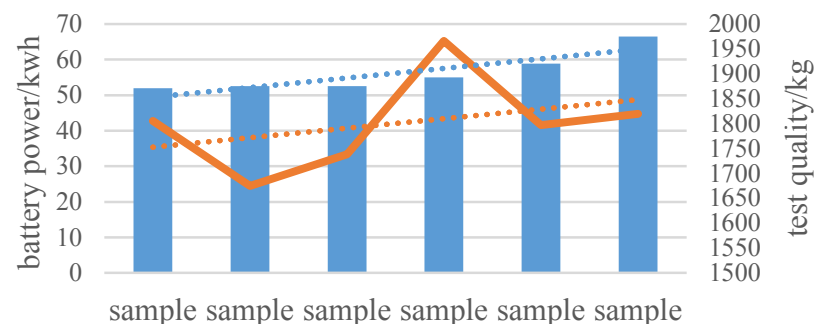

$\begin{array}{llllll}1 & 2 & 3 & 4 & 5 & 6\end{array}$

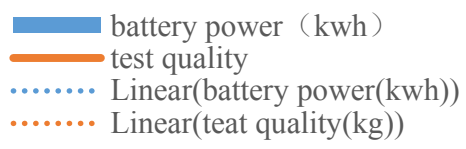

Fig. 4. Relationship between battery power and test quality.

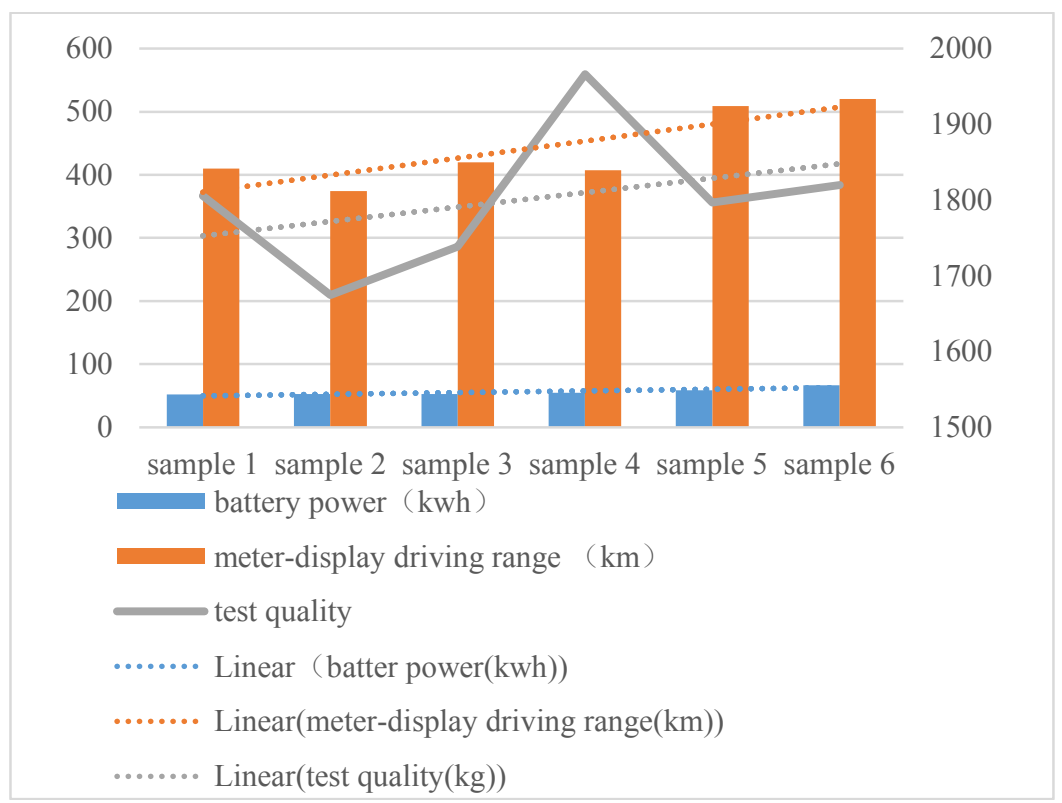

Fig. 5. Relationship between battery power, test quality and meter-display driving range.

From the diagram in Fig .4 and 5, we can see that the size of the test quality increases when the battery power increase, which effects the driving range of electric vehicles. But it's not that the bigger the battery, the more the driving range increases.

\section{Test results and analysis}

\subsection{Analysis of driving range at normal temperature}

This test uses different test conditions CLTC-P rather than others in the past, which conform to the actual conditions of Chinese roads and are more accurate than the NEDC conditions test used in the past. The comparison results between the test data of the selected 6 vehicles 
at normal temperature and the driving range of the vehicle displayed at normal temperature are shown in Figure 6 below:

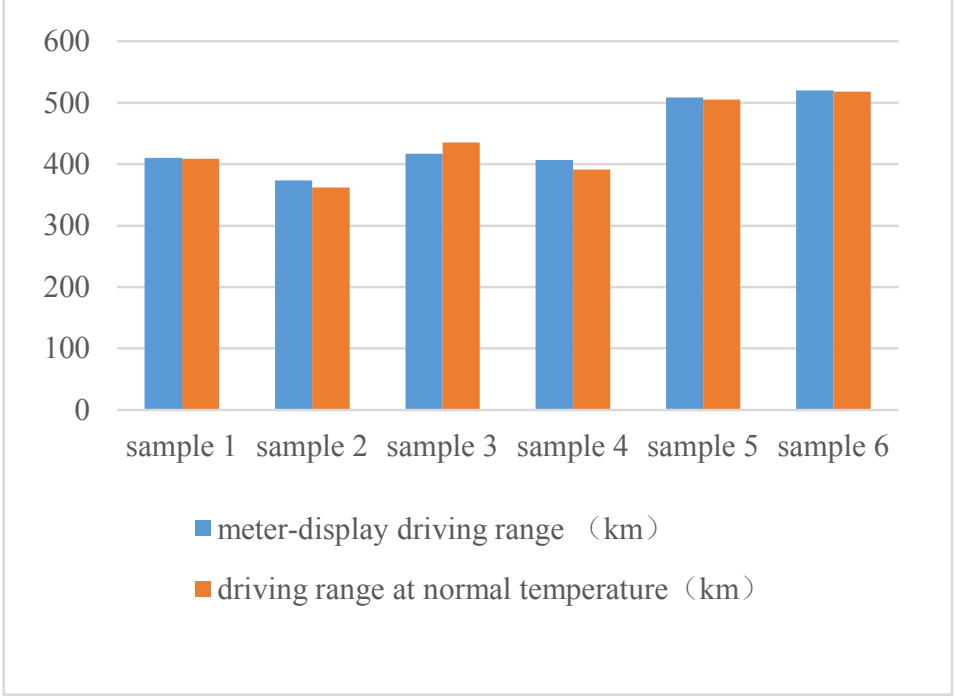

Fig. 6. Comparison between the driving range at normal temperature and the meter-display driving range.

From the figure, we can see that the test results of the continuous driving range of the 6 test vehicles at normal temperature are not much different from the displayed driving range. The reason why the driving range at normal temperature of the sample car 3 is higher than the initial value of the display is that the sample car's braking energy recovery level is higher than that of several other cars. And there are many curve segments that need to be braked in the CLTC-P test conditions. The initial value of the display is calibrated according to the driving range of NEDC.

The displayed remaining range is the most intuitive number that the driver can see when driving, which means that when commuting to work without using air conditioning and other on-board electrical equipment, the remaining range displayed on the dashboard can be used to judge the the remaining battery life of electric vehicle, so as to perform the necessary charging.

\subsection{Analysis of driving range at normal temperature for high-speed $(100 \mathrm{~km} /$ h)}

The difference between the normal temperature endurance test and high-speed test is that the vehicle speed is adjusted according to the operating condition curve during the normal temperature endurance test. During braking, a part of the braking energy recovery is set according to the different vehicle. But in the high speed test, the vehicle has been at a steady and high speed. The battery is fully discharged and there is no braking energy recovery, and the driving range will be relatively reduced. However, the driving range at normal temperature is very close to the driving range of the meter displayed, so we can also determine the approximate remaining driving range of the high-speed by the displayed driving range. The drop rate of normal temperature high-speed driving range relative to the meter-display driving range is shown in Figure 7: 


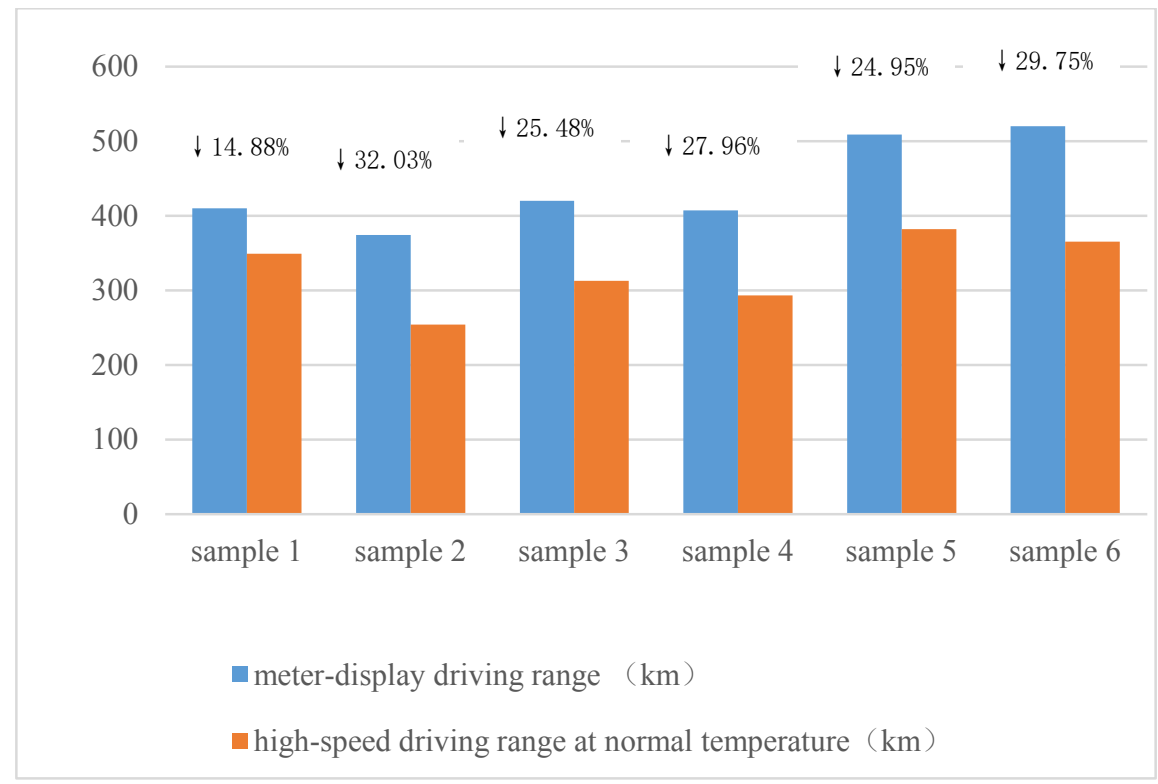

Fig. 7. Drop rate of high-speed cruising range at normal temperature.

It can be seen from the comparison between the results of the high-speed continuous driving range test at normal temperature, the decline rate of most vehicles remains within the range of $25 \%-35 \%$.

The comparison chart of the rate of decline between the high-speed continuous driving range at normal temperature and the meter-displayed continuous driving range and normal temperature continuous driving range is shown in Figure 8 :

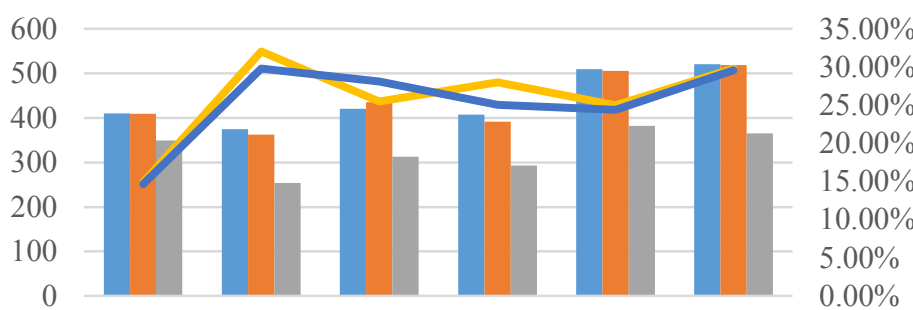

sample 1 sample 2 sample 3 sample 4 sample 5 sample 6

meter-display driving range $(\mathrm{km})$

driving range at normal temperature $(\mathrm{km})$

high-speed driving range at normal temperature $(\mathrm{km})$

Decline rate of relative to meter-display driving range

Decline rate of relative to normal temperatrue driving range

Fig. 8. Comparison of decline rate.

As can be seen from the figure that the drop rates of sample car 1, sample car 5 and sample car 6 are almost close. The drop rate of sample car 2 is the highest because the most economical driving mode is selected during the normal temperature endurance test When 
the maximum speed cannot reach the maximum speed requirement of CLTC-P, the driving mode that can keep up with the test operating curve is selected. This mode consumes more power than the most economical ECO mode. In order to ensure consistency, the same driving mode is adopted in the high-speed endurance test and tested at normal temperature. The drop rate of the sample car 3 was greatly different because the higher braking energy recovery level during the normal temperature endurance test made the continuous driving range at normal temperature longer than the displayed distance. The difference of the drop rate of sample car 4 is caused by the highest test quality of the sample car, so the test result of normal temperature endurance is lower than that of the driving range of the vehicle displayed.

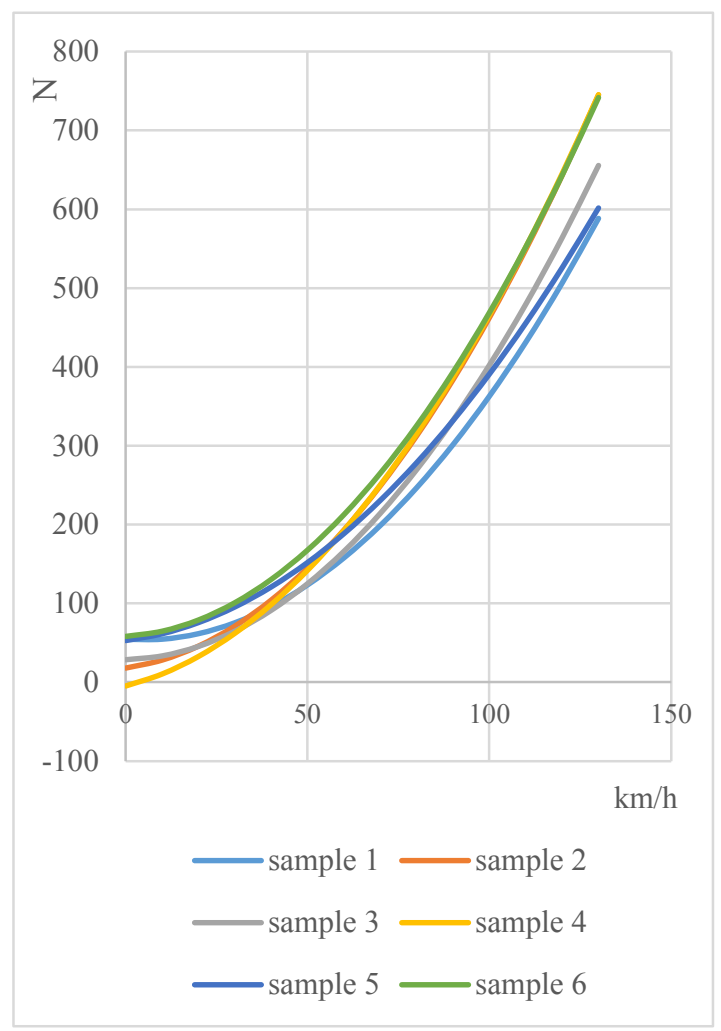

Fig. 9. Comparison of chassis dynamometer resistance.

From the comparison chart of the chassis dynamometer resistance in Figure 9, it can be seen that the external resistance experienced by the test vehicle at a speed of $100 \mathrm{~km} / \mathrm{h}$ is sorted from largest to smallest: sample car 6-sample car 4- sample car 2- sample car 3sample car 5-sample car 1 . The decreasing rate of high-speed driving range relative to normal temperature driving range is as follows: sample car 2-sample car 6-sample car 3-sample car 4-sample car 5-sample car 1. Due to the different driving mode of sample car 2 , it is normal for the descent rate to be high. On the whole, it can be roughly judged that the external resistance received by the vehicle will also partially affect the high-speed driving range.

The overall test results are different. On the one hand, the quality of each car is different, which will effect the external resistance of the vehicle; on the other hand, the battery performance and distribution strategies and so on of different vehicles will have some influence on the result. Furthermore, the choice of driving mode will have a great influence on the test results. 


\title{
3. Conclusion
}

\begin{abstract}
Above all, the research of the electric vehicle's normal temperature $\left(25^{\circ} \mathrm{C}\right)$ high-speed endurance is compared from three aspects: meter-display driving range, normal temperature driving range and normal temperature high-speed driving range. It is concluded that the drop rate of normal temperature high-speed driving range for the meter-display driving range and normal temperature driving range is between $25 \%$ and $35 \%$. Increasing the battery power can extend the normal temperature driving range and normal temperature high-speed driving range to a some extent, but the quality of the vehicle itself will also increase to reduce the role of battery power. So it is not linear to increase battery power and extend high-speed driving range. In future design of vehicles should increase battery power while achieving lightweight design of battery and body, as well as streamlined design of the body to reduce the external resistance of the vehicle during driving. The driver keeps a gentle acceleration as far as possible while driving the vehicle in order to reduce the acute acceleration reduction, which will appropriately increase some driving range. Similarly, choosing the most economical driving mode and the highest energy recovery level while driving can effectively improve the driving range of the vehicle, but this will reduce part of the driving experience.
\end{abstract}

\section{References}

1. GB/T 19596-2017 Terminology for electric vehicles, in Chinese

2. GB / T 20234.1-2015 Connection device for conductive charging of electric vehicles Part 1 General requirements, in Chinese

3. GB / T 18386-2005 Test Method for Energy Consumption Rate and Driving Range of Electric Vehicles, in Chinese

4. GB 18352.6-2016 Limits and Measurement Methods of Light Vehicle Pollutants Emission (China Sixth Stage), in China

5. GB/T 12534 General rules of automobile road test methods, in Chinese

6. EV-TEST Electric Vehicle Evaluation Management Rules (2019 Edition). China Automotive Technology Research Center Co., Ltd

7. Chan C C.The state of the art of electric, hybrid, and fuel cell vehicles[J].Proceedings of the IEEE, 2007,95(4):704-718.

8. XieNan, Experimental study on the influence of ambient temperature on the driving range of electric vehicles, Journal of Chongqing University of Technology (Natural Science), in Chinese

9. Zhang Shuqiao, Development History, Current Situation and Manufacturing Technology of New Energy Electric Vehicles, Metal Processing (Cold Processing), in Chinese

10. Wang Zhenchao, Research on the Location Selection of Electric Vehicle Charging Piles in the City, Science and Technology Economics, in Chinese 